\title{
Abordagem fisioterapêutica no pré-parto: proposta de protocolo e avaliação da dor
}

\author{
Physiotherapeutic approach in the pre-partum: proposed protocol and evaluate pain \\ Amanda de Souza Castro', Ana Carolina de Castro', Adriana Clemente Mendonça²
}

RESUMO I O objetivo deste estudo foi avaliar os efeitos da abordagem fisioterapêutica no pré-parto e propor um protocolo de intervenção baseado na escala visual analógica (EVA) de dor. Dez parturientes, na primeira fase do trabalho de parto, foram questionadas quanto à dor através da EVA e, com base nas respostas, foi proposto um protocolo, EVA 1-3: cinesioterapia, técnicas respiratórias, relaxamento e estímulo à deambulação; EVA 4-7: massoterapia, técnicas respiratórias, relaxamento e estímulo à deambulação; EVA 8-10: técnicas respiratórias, relaxamento e eletroestimulação nervosa transcutânea. Após as intervenções, as voluntárias foram questionadas novamente através da EVA. Os dados da EVA pré- e pós-intervenção foram submetidos ao teste $t$ pareado, atingindo valor médio de 8,8 pré-intervenção e 8,2 pós-intervenção, sem diferença estatística. Os resultados obtidos por meio da análise quantitativa de dor demonstraram que não houve aumento da mesma até uma hora após a intervenção, fato considerado positivo, uma vez que em função da crescente dilatação é esperado um aumento da dor. O protocolo fisioterapêutico proposto mostrou ser de fácil aplicabilidade, podendo auxiliar o fisioterapeuta na escolha da conduta mais adequada à realidade da sala de pré-parto. A abordagem fisioterapêutica no pré-parto parece interferir positivamente sobre a dor e o desconforto materno no grupo estudado.

Descritores I fisioterapia; gestantes; trabalho de parto; dor; saúde da mulher.
ABSTRACT I The aim of this study was to evaluate the effects of physical therapy approach in the antepartum and to propose an intervention protocol based on the visual analogue scale (VAS) of pain. Ten parturients in the first stage of labor were questioned about the pain by VAS. Based on the responses, we proposed a protocol VAS 1-3: kinesiotherapy, breathing techniques, relaxation and stimulation of walking; VAS 4-7: massage therapy, breathing techniques, relaxation and stimulation of walking; VAS 8-10: breathing techniques, relaxation and transcutaneous electrical stimulation. After the intervention, the volunteers were questioned again by VAS. The VAS data preand post-intervention were submitted to the paired $t$ test, reaching an average value of 8.8 pre-intervention and 8.2 post-intervention, no statistical difference. The results obtained through quantitative analysis of pain showed no increase of the same until one hour after the intervention, which was considered positive, since due to the increasing expansion is expected to increase the pain. The proposed physical therapy protocol proved easy to apply. It can assist the therapist in choosing the most adequate to the reality of predelivery room. The physical therapy approach in the pre-partum seems to positively affect pain and maternal discomfort in the study group.

Keywords I physical therapy specialty; pregnant women; labor, obstetric; pain; women's health.

Estudo desenvolvido no Curso de Fisioterapia do Hospital de Clínicas da Universidade Federal do Triângulo Mineiro (UFTM) - Uberaba (MG), Brasil.

'Fisioterapeura pela UFTM - Uberaba (MG), Brasil.

2Fisioterapeuta; Doutora em Ciências Médicas pela Faculdade de Medicina de Ribeirão Preto da Universidade de São Paulo (USP) Ribeirão Preto (SP), Brasil; Docente do curso de Fisioterapia da UFTM, laboratório de Reabilitação e Qualidade de Vida (REQUALI) Uberaba (MG), Brasil. 


\section{INTRODUÇÃO}

O trabalho de parto é dividido em duas fases: a primeira é caracterizada por contrações uterinas que permitem a dilatação progressiva do colo uterino. Trata-se de uma fase em que o estresse emocional está presente, bem como a dor; a segunda fase corresponde à expulsão fetal, na qual as contrações e a dilatação do colo uterino se tornam mais intensas ${ }^{1}$.

A evolução do trabalho de parto é mensurada pela cervicodilatação uterina, por meio do toque vaginal. A progressão da dilatação cervical acelera-se a partir dos $4 \mathrm{~cm}$, caracterizando a fase ativa do trabalho de parto, quando a velocidade da dilatação cervical se dá em torno de $1 \mathrm{~cm}$ por hora ${ }^{2}$.

Quando se inicia o trabalho de parto, é comum que se sintam dores provenientes das contrações, que estão presentes tanto na primeira, quanto na segunda fase. A dor sentida pela mulher nesse período pode sofrer influências socioculturais, psicológicas, da individualidade e do ambiente em que são atendidas, e é impossível de ser compreendida por quem não a está experimentando, devendo ser respeitada e nunca subestimada $^{3-5}$.

Os programas multidisciplinares de preparação para o parto vêm sendo desenvolvidos a fim de proporcionar à parturiente o equilíbrio físico e psíquico, e a sensação de bem-estar. Esses programas têm como benefícios a diminuição dos sintomas de desconforto e dor do parto, controle da ansiedade, diminuição do tempo de trabalho de parto e do índice de indicação para parto cesárea ${ }^{6-9}$.

Dentre os membros da equipe multidisciplinar, encontra-se o fisioterapeuta, que tem como função avaliar e monitorar as alterações físicas enfocando a manutenção do bem-estar da parturiente e do bebê, tanto na primeira quanto na segunda fase do trabalho de parto $^{1,7,8,10}$.

No desempenho dos cuidados com as parturientes, é recomendada a adoção de tecnologias não farmacológicas e não invasivas para o alívio da dor ${ }^{3,9,11}$, dentre elas podemos citar a eletroestimulação nervosa transcutânea (TENS), a hidroterapia, a cinesioterapia, a crioterapia, a massoterapia, as técnicas respiratórias e de relaxamento ${ }^{6,9,12-18}$.

Este estudo teve como objetivo avaliar os efeitos da abordagem fisioterapêutica sobre a dor da parturiente na primeira fase do trabalho de parto, bem como propor um protocolo de intervenção baseado na escala visual analógica (EVA) de dor.

\section{METODOLOGIA}

\section{Participantes}

Fizeram parte deste estudo 10 parturientes, entre 18 e 30 anos ( $\pm 22,8$ anos), internadas no setor de Ginecologia e Obstetrícia do Hospital de Clínicas da Universidade Federal do Triângulo Mineiro (UFTM). Para serem incluídas, as voluntárias precisavam estar na primeira fase do trabalho de parto, apresentar dor e não estarem sob efeito de analgésico. Foram excluídas menores de 18 anos e gestação de risco. Este estudo foi aprovado pelo Comitê de Ética em Pesquisa da UFTM (Protocolo no 1.387). Após o aceite em participar, as voluntárias leram e assinaram o termo de consentimento livre e esclarecido.

\section{Instrumentos e procedimentos}

Foi elaborada uma ficha de avaliação com questões sobre os dados pessoais das participantes, bem como dados gestacionais (gestações anteriores, pré-natal, intercorrências), doenças associadas, e sobre a caracterização da dor na primeira fase do trabalho de parto. A avaliação da dor foi realizada através da EVA, antes e após a intervenção fisioterapêutica (uma hora após), e foi elaborado um protocolo de tratamento que levou em consideração a intensidade da dor referida pelas voluntárias. O tempo máximo de intervenção fisioterapêutica foi de $50 \mathrm{minu}-$ tos, sendo realizada uma única vez em cada voluntária.

\section{Protocolo de intervenção fisioterapêutica}

Este protocolo foi elaborado com base na realidade da sala de pré-parto em nosso serviço e levou em consideração o nível de dor de acordo com a EVA.

EVA (1-3): cinesioterapia, técnicas respiratórias, relaxamento e estímulo à deambulação; EVA (4-7): massoterapia, técnicas respiratórias, relaxamento e estímulo à deambulação e EVA (8-10): técnicas respiratórias, relaxamento e TENS. Para cada recurso terapêutico foram selecionadas as seguintes técnicas:

- Cinesioterapia: realizada através de exercícios com bola e bastão, exercícios de retroversão e anteversão pélvica, exercícios ativos de membros superiores e inferiores, deambulação associada a exercícios de membros superiores e respiratórios;

- Massoterapia: realizada com técnicas de deslizamentos superficiais e suaves, com cremes ou óleos, com a gestante posicionada sentada ou deitada em decúbito lateral esquerdo; 
- Técnicas respiratórias e relaxamento: realizadas com incursões inspiratórias e expiratórias prolongadas, propriocepção diafragmática, incluindo comandos verbais e orientações para preparação ao parto;

- TENS: utilizada a técnica tetrapolar cruzada na região lombar, com os parâmetros para dor aguda, ou seja, frequência elevada $(150 \mathrm{~Hz})$ e duração de pulso moderada (75 $\mu \mathrm{s})$, com tempo de aplicação de 30 minutos. Os eletrodos foram posicionados na região dos dermátomos de T10 e L1, que correspondem à inervação do útero e cérvix, respectivamente.

\section{Análise estatística}

Os dados quantitativos da dor avaliados na pré- e pós-intervenção fisioterapêutica foram submetidos ao teste de normalidade (ShapiroWilk) e, depois de constatada sua normalidade, foram avaliados por meio do teste $t$ pareado, sendo considerado significativo quando $\mathrm{p}<0,05$.

\section{RESULTADOS}

Todas as voluntárias deste estudo realizaram pré-natal, apenas $40 \%$ realizaram mais de 7 consultas e nenhuma delas realizou fisioterapia durante a gestação. Dessas, $60 \%$ estavam entre a $35^{\text {a }}$ e $38^{a}$ semana, e $40 \%$ entre a $39^{a}$ e $42^{a}$ semana, $50 \%$ eram primigestas e $50 \%$ multípara, com prevalência de partos normais.

Setenta por cento estavam em uso de ocitocina para induzir o trabalho de parto. A dinâmica uterina da amostra variou entre 2 contrações com duração de 40 segundos a cada 10 minutos e 5 contrações com duração de 50 segundos a cada 10 minutos. Quanto à dilatação uterina, $40 \%$ apresentavam de 1 a $4 \mathrm{~cm}$, e $60 \%$ de 5 a $8 \mathrm{~cm}$.

A dor referida pelas voluntárias estava localizada em baixo ventre (30\%), em baixo ventre com irradiação para a coluna lombar (60\%) e somente na coluna lombar (10\%).

Durante a avaliação inicial, a voluntária foi questionada quanto à posição em que ocorria diminuição da dor e a maioria (70\%) relatou ser em decúbito lateral, $10 \%$ durante a deambulação e $20 \%$ relatou que a dor não amenizava em nenhuma posição.

O nível de dor avaliado pela EVA está representado na Figura 1, onde a média antes do tratamento foi de $8,8( \pm 1,39)$ e após o tratamento $8,2( \pm 1,93)$, sem diferença estatística $(p=0,07)$.

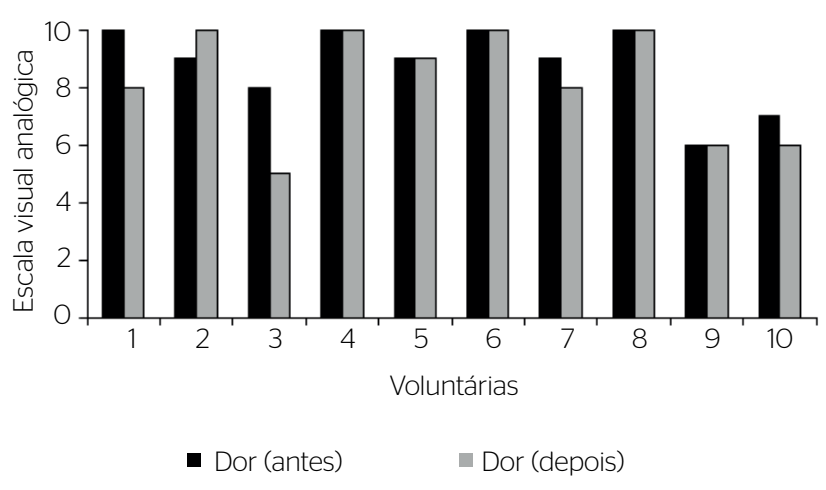

Figura 1. Nível de dor na escala visual analógica mensurada antes e após uma hora da intervenção fisioterapêutica

\section{DISCUSSÃO}

Embora os resultados quantitativos da avaliação da dor não tenham demonstrado diferenças significativas após intervenção fisioterapêutica, o simples fato dessa dor não ter aumentado nesse período de aproximadamente duas horas (período gasto entre a avaliação, intervenção e nova coleta) demonstra resultados benéficos da abordagem fisioterapêutica nessa fase, pois sabemos que a dor é devido à dilatação do colo uterino, portanto aumentando proporcionalmente ao mesmo.

Os valores de dilatação nesse período de 2 horas foram relativamente altos, entre 1 e $4 \mathrm{~cm}$ em 4 voluntárias e entre 5 e $8 \mathrm{~cm}$ em 6 voluntárias. $\mathrm{O}$ simples aumento da dilatação uterina deveria aumentar o nível de dor, mas isso só ocorreu na voluntária 2, sendo que esta foi a parturiente que apresentou o maior valor de dilatação $(8 \mathrm{~cm})$, o que poderia justificar esse aumento da dor.

Foi observada em todas as voluntárias uma melhora qualitativa, difícil de ser mensurada; sinais, como diminuição da ansiedade, do estresse materno e aumento da segurança experimentada pela gestante nesse período, foram relatados pelas mesmas na visita realizada após o nascimento do bebê. A equipe médica e de enfermagem também sentiu grande diferença no comportamento das parturientes que eram atendidas na sala de pré-parto pela fisioterapia, quando comparadas àquelas que não recebiam o atendimento, interferindo inclusive no tempo de evolução para o parto.

Esses achados vão de encontro àqueles apresentados por Almeida et al. ${ }^{6}$ que concluíram, após intervenção física em parturientes, que não ocorria redução da intensidade da dor, porém diminuía o nível de sua ansiedade. O controle da ansiedade durante o trabalho de parto é 
fundamental, pois quanto maior a ansiedade, maior o nível de dor relatado pela parturiente ${ }^{19,20}$.

A indução do trabalho de parto é praticada sistematicamente apenas em alguns poucos hospitais, geralmente públicos e universitários, como no caso deste estudo. Esse é um procedimento aceitável e recomendável sob o ponto de vista médico e humano, sempre que exista uma indicação para isso. No grupo estudado, 70\% das voluntárias estavam em trabalho de parto induzido por ocitocina, que é o método mais utilizado e conhecido para a indução do parto vaginal ${ }^{21}$. Sete voluntárias evoluíram para parto normal e três para cesariana.

A dor, durante o trabalho de parto, é uma resposta fisiológica, complexa, subjetiva e multidimensional aos estímulos sensoriais gerados, principalmente, pela contração uterina ${ }^{6,22}$. As contrações uterinas são dolorosas por causas não totalmente estabelecidas, como hipóxia do miométrio contraído, compressão de gânglios nervosos na cérvix e na parte inferior do útero pelos feixes musculares entrelaçados, estiramento cervical durante a dilatação e estiramento do peritônio subjacente ao fundo uterino ${ }^{23}$.

Assim, observa-se a necessidade de aliviar a dor e a ansiedade da parturiente, uma vez que esses mecanismos podem prejudicar o feto e afetar a progressão fisiológica do trabalho de parto. $\mathrm{O}$ uso de métodos não farmacológicos no alívio da dor durante o trabalho de parto tem a vantagem de reduzir e/ou postergar o uso de fármacos no controle da dor, proporcionando condições para a colaboração ativa da mulher e permitindo maior participação do acompanhante ${ }^{23}$.

A característica e a localização da dor encontradas neste estudo estão de acordo com a literatura, que a descreve como cólica abdominal, incômodo, e desconforto baixo, expressando excitação e apreensão, podendo se localizar no centro das costas e no abdome anterior, sendo referida como intensa, constritiva e frequentemente excruciante ${ }^{24}$.

A posição em decúbito lateral promove alívio da dor e beneficia a circulação uteroplacentária, além de reduzir a duração do trabalho de parto ${ }^{1,25}$. Essa afirmação explica o que foi relatado pelas voluntárias deste estudo. Contudo, estudos relatam que a melhor posição para a parturiente assumir é aquela em que ela se sente mais confortável. Logo, ela pode tanto andar como deitar, sentar com apoio nas costas, ou apenas inclinar o corpo para frente com algum tipo de sustentação ${ }^{25}$.

O uso da TENS no alívio da dor, tanto no préquanto no pós-parto já está amplamente divulgado e estudos demonstram que esse recurso é capaz de aliviar as tensões musculares e diminuir a dor por quebra do ciclo dor/espasmo/dor ${ }^{14,26-28}$. Embora, em recente revisão sistemática, os resultados tenham sido inconclusivos quanto ao efeito da TENS no controle da dor do parto quando comparado ao do grupo placebo, os autores relatam ainda uma baixa qualidade metodológica na maioria dos estudos incluídos ${ }^{29}$.

Não foi objetivo deste trabalho comparar as diferentes técnicas fisioterapêuticas aplicadas, mas sim a abordagem como um todo. Daí a proposta deste protocolo, pois se observa uma grande resistência das parturientes na primeira fase do trabalho de parto em realizar exercícios e deambulação, quando os valores de dor mensurados pela EVA encontravam-se altos. Salientamos a importância da deambulação nessa fase, pois essa técnica melhora a evolução da dilatação, diminuindo a duração da fase ativa ${ }^{10,12,15,30}$. Portanto, a deambulação deve ser sempre estimulada, pois favorece a descida fetal e antecipa o trabalho de parto, no entanto deve-se sempre levar em consideração a queixa da paciente, respeitando seu limiar doloroso e aceitação da intervenção proposta. Para Porto et al. ${ }^{31}$, a deambulação ou o posicionamento mais confortável escolhido pela parturiente devem ser adotados, sempre previamente discutidos com as mesmas.

Neste estudo, as voluntárias não apresentaram boa aceitação para deambular quando a dor estava intensa, por isso a sugestão do protocolo sem a inclusão da deambulação com valores entre 8 e 10 da dor na EVA. Dentre as voluntárias deste estudo, nenhuma apresentou dor entre 1 e 3-EVA, mas em função da aceitação das voluntárias com dor entre 4 e 7-EVA à deambulação precedida de massagem, espera-se que com um nível menor de dor, a parturiente permita a realização do protocolo sem a necessidade da massagem. No entanto, por se tratar de uma amostra pequena, se faz necessário um maior número de estudos para adequação de protocolos com base na realidade das salas de pré-parto.

A inserção do fisioterapeuta na equipe inderdisciplinar atuando tanto na primeira, quanto na segunda fase do trabalho de parto, ainda é pequena e em um trabalho de revisão da literatura os autores relataram que a atuação fisioterapêutica durante o trabalho de parto parece favorecê-lo, mas há estudos que não mostraram diferenças entre eles, o que dificulta definir técnicas específicas ${ }^{8}$.

\section{CONCLUSÃO}

O protocolo fisioterapêutico proposto neste estudo, com base na EVA de dor mostrou ser de fácil aplicabilidade, 
podendo auxiliar o fisioterapeuta na escolha da conduta mais adequada à realidade da sala de pré-parto. A abordagem fisioterapêutica no pré-parto parece interferir positivamente sobre a dor e o desconforto materno no grupo estudado, porém novos estudos se fazem necessários nessa área.

\section{AGRADECIMENTOS}

Agradecemos a colaboração das parturientes e da equipe multiprofissional de assistência ao parto do Hospital de Clínicas da UFTM.

\section{REFERÊNCIAS}

1. Baracho E. Fisioterapia aplicada à obstetrícia, uroginecologia e aspectos de mastologia. 4a ed. Rio de Janeiro: Guanabara Koogan; 2007.

2. Brasil. Ministério da Saúde, Secretaria de Políticas de Saúde. Area Técnica de Saúde da Mulher. Manual puérperio. Brasília; 2006. 162 p.

3. Macedo PO, Progianti JM, Vargens OMC, Santos VLC, Silva CA. Percepção da dor pela mulher no pré-parto: a influência do ambiente. Rev Enferm UERJ. 2005:3(3):306-12.

4. Davim RMB, Torres GV, Dantas, JC. Representação de parturientes acerca da dor de parto. Rev Eletr Enf. 2008;10(1):100-9.

5. Cunha AA. Analgesia e anestesia no trabalho de parto e parto. Femina. 2010;38(11):599-606

6. Almeida NAM, Sousa JT, Bachion MM, Silveira NA. Utilização de técnicas de respiração e relaxamento para alívio de dor e ansiedade no processo de parturição. Rev Latino-am Enfermagem. 2005:13(1):52-8.

7. Bio E, Bittar RE, Maganha CA, Zugaib M. Intervenção fisioterapêutica no trabalho de parto. Femina. 2005;33(10):783-7.

8. Canesin KF, Amaral, WN. Atuação fisioterapêutica para diminuição do tempo do trabalho de parto: revisão de literatura. Femina. 2010;38(8)429-33.

9. Gallo RBS, Santana LS, Marcolin AC, Ferreira CHJ, Duarte G, Quintana SM. Recursos não-farmacológicos no trabalho de parto: protocolo assistencial. Femina. 2011;39(1):41-8.

10. Bavaresco GZ, Souza RS, Almeica B, Sabatino JH, Dias M. O fisioterapeuta como profissional de suporte à parturiente. Ciênc Saúde Coletiva. 2011:16(7):3259-66.

11. Sescato AC, Souza SRRK, Wall ML. Os cuidados não-farmacológicos para alívio da dor no trabalho de parto: orientações da equipe de enfermagem. Cogitare Enferm. 2008:13(4):585-90.

12. Bio E, Bittar RE, Zugaib M. Mobilidade materna na duração da fase ativa do trabalho de parto. Rev Bras Ginecol Obstet. 2006;28(11):671-9.
13. Bõing I, Sperandio FF, Santos GM. Uso de técnica respiratória para analgesia no parto. Femina. 2007;35(1):41-6.

14. Knobel R, Radunz V. Carraro TE. Utilização de estimulação elétrica transcutânea para alívio da dor no trabalho de parto: um modo possível para o cuidado à parturiente. Texto Contexto - Enferm. 2005;14(2):229-36.

15. Mamede FV, Almeida AM, Nakano MAS, Gomes FA, Panobianco MS. O efeito da deambulação na duração da fase ativa do trabalho de parto. Rev Latino-am Enfermagem. 2007;11(3):466-71.

16. Nunes S, Vargens OMC. A crioterapia como estratégia para alívio da dor no trabalho de parto: um estudo exploratório. Rev Enferm UERJ. 2007:15(3):337-42

17. Mazoni SR, Faria DGS, Manfredo VA. Hidroterapia durante o trabalho de parto: relato de uma prática segura. Arq Ciênc Saúde. 2009:16(1):40-3.

18. Silva LM, Oliveira SMJV, Silva FMB, Alvarenga MB. Uso da bola suiça no trabalho de parto. Acta Paul Enferm. 2011;24(5):656-62.

19. Curzik D, Jokic-Begic N. Anxiety sensitivity and anxiety as correlates of expected, experienced and recalled labor pain. J Psychosom Obstet Gynaecol. 2011;32(4):198-203.

20. Veringa I, Buitendijk S, de Miranda E, de Wolf S, Spinhoven P. Pain cognitions as predictors of the request for pain relief during the first stage of labor: a prospective study. J Psychosom Obstet Gynaecol. 2011;32(3):119-25.

21. Moraes Filho OB, Cecatti JG, Feitosa FEL. Métodos para indução do parto. Rev Bras Ginecol Obstet. 2005;27(8):493-500.

22. Lowe NK. The nature of labor pain. Am J Obstet Gynecol. 2002;186(5 Suppl Nature):S16-24

23. Silva FMB, Oliveira SMJV. O efeito do banho de imersão na duração do trabalho de parto. Rev Esc Enf USP. 2006;40(1):57-63.

24. Davim RMB, Torres GV, Melo ES. Estratégias não farmacológicas no alívio da dor durante o trabalho de parto: pré-teste de um instrumento. Rev Latino-am Enfermagem. 2007:15(6):1150-6.

25. Mazzali L, Gonçalves RN. Análise do tratamento fisioterapêutico na diminuição da dor durante o trabalho de parto. Ensaios e Ciência: C Agrárias, Biológicas e da Saúde. 2008;12(1):7-17.

26. Orange FA, Amorim MMR, Lima L. Uso da eletroestimulação transcutânea para alívio da dor durante o trabalho de parto em uma maternidade-escola: ensaio clínico controlado. Rev Bras Ginecol Obstet. 2003;25(1):45-52.

27. Tribioli RA. Estimulação elétrica nervosa transcutânea durante o trabalho de parto. Femina. 2005:33(11):865-8.

28. Telles ER, Amaral VF. Estimulação elétrica transcutânea (TENS) em ginecologia e obstetrícia: alternativa nas síndromes dolorosas. Femina. 2007;35(11):697-702

29. Mello LF, Nóbrega LF, Lemos A. Transcutaneous electrical stimulation for pain relief during labor: a systematic review and meta-analysis. Rev Bras Fisioter. 2011:15(3):175-84.

30. Amorim MMR, Porto AMF, Souza ASR. Assistência ao segundo e terceiro períodos do trabalho de parto baseada em evidências. Femina. 2010;38(11):583-91.

31. Porto AMF, Amorim MMR, Souza ASR. Assistência ao primeiro período do trabalho de parto baseada em evidências: [revisão]. Femina. 2010;38(10)527-37. 